\title{
Sulforaphane Inhibits Self-renewal of Lung Cancer Stem Cells Through the Modulation of Sonic Hedgehog Signaling Pathway and Polyhomeotic Homolog3
}

\author{
FanPing Wang \\ Xinxiang Medicall University \\ Yanwei Sun \\ Xinxiang Medical University \\ Xiaoyu Huang \\ Xinxiang Medical University \\ Caijuan Qiao \\ Xinxiang Medical University \\ Wenrui Zhang \\ Xinxiang Medical University \\ Peijun Liu \\ Xi'an Jiaotong University \\ Mingyong Wang ( $\nabla$ wmy118@126.com) \\ Xinxiang Medical University
}

Original article

Keywords: Sulforaphane, Lung cancer, Cancer stem cell, Sonic Hedgehog Signaling Pathway, Human Polyhomeotic Homolog 3

Posted Date: February 4th, 2021

DOl: https://doi.org/10.21203/rs.3.rs-165086/v1

License: (9) This work is licensed under a Creative Commons Attribution 4.0 International License. Read Full License 


\section{Abstract}

Sulforaphane (SFN), an active compound in cruciferous vegetables has been characterized for its antiproliferative capacity. We investigated the role and molecular mechanism through which SFN regulates proliferation and self-renewal of lung cancer stem cells (CSCs). CD133-positive lung cancer cells were isolated by MACs from lung cancer A549 and H460 cells. And then, the expression of CD133 was measured by flow cytometry assays (FACS). The ability of cell proliferation was assessed by MTT assays and tumorsphere formation assays. The mRNA expression of Sonic Hedgehog (Shh), Smoothened (Smo), Gli1 and Human Polyhomeotic Homolog 3 (PHC3) was measured by quantitative reverse transcription polymerase chain reaction (QPCR). And the protein expression of Shh, Smo, Gli1 and PHC3 was determined by western blotting. Shh was knocked down by siRNA to further study the role of Shh signaling pathways in lung CSCs. SFN inhibited the proliferation of lung cancer cells and lung CSCs simultaneously. Meanwhile, the mRNA and protein expressions of Shh, Smo, Gli1 and PHC3 were highly activated in A549 /CD133+ and H460 /CD133+ cells. Compared with siRNA-control group, Knock-down of Shh inhibited proliferation of A549/ CD133+ and H460/ CD133+ cells, and decreased the protein expression of PHC3 in A549/ CD133+ and H460/ CD133+ cells. In addition, SFN inhibited the activities of Shh, Smo, Gli1 and PHC3 in A549/ CD133+ and H460/ CD133+ cells. Furthermore, the inhibitory effect of SFN on the proliferation of siRNA-shh cells is weaker than that of siRNA-control cells. SFN is an effective new drug which can inhibit proliferation of lung CSCs through the modulation of PHC3 and SHH signaling pathways. It provides a novel target for improving therapeutic efficacy for lung CSCs.

\section{Introduction}

Lung cancer is one of the most prevalent cancers which accounts for approximately one-fourth of the cancer incidence and is the second leading cause of death both in China and developed countries around the world (Torre et al. 2016; Siegel et al. 2019). Despite initial treatment with conventional therapy has a high remission rate, eventually the disease almost always relapses in the form of resistance to chemotherapy and radiation therapy, therefore the overall 5-year survival rate of patients is less than $5 \%$ ( Bray et al. 2018).

Increasing evidence of the existence of cancer stem cells(CSCs) in lung cancer explains why standard chemotherapy or radiotherapy regimens against lung cancer are usually ineffective and result in further tumor recurrence and spread (Hermann et al. 2010). CD133 is a cell membrane glycoprotein and it contains five transmembrane rings. It has been reported that CD133-positive subpopulation of multipotent cells has the biological features of CSCs which possess extremely proliferative and selfrenewal characteristics (Kim et al. 2017; Mizugaki et al. 2014). Although CSCs are only a small part of tumors, they have a powerful ability to self-renew and divide. The tumors rich in CSCs are more aggressive and lead to worse clinical outcomes. Therefore, the development of therapeutic strategies and drugs that specifically target CSCs can eradicate tumors effectively and reduce the risk of recurrence and metastasis. 
Sulforaphane (SFN) is an isothiocyanate (ITC) found in cruciferous vegetables such as broccoli and cabbage (Clarke et al. 2008; Sangthong et al. 2016). A series of clinical trials have demonstrated that SFN can inhibit the malignant growth of cancer cells such as pancreatic cancer cells and breast cancer cells and has no obvious toxicity to the normal cells (Fisher et al. 2017; Jaman et al. 2018; Srivastava et al. 2011; Subramaniam et al. 2018). Therefore, it has been suggested safe to use SFN as a potential candidate for cancer treatment. However, the effect of SFN on the development of lung cancer and its mechanism is still unknown.

Sonic Hedgehog $(\mathrm{SHH})$ signaling pathway has an essential role in the control of stem cell growth in embryonic tissues, and it plays a key role in the development of tissues and organs (Varjosalo et al. 2008). Recent evidence suggests that the SHH signaling pathway contributes to tumorigenesis when it is mutated or misregulated (Subramani et al. 2017; Song et al. 2017). Since the SHH pathway plays a critical role in the renewal of CSCs, blockade of SHH pathway has evolved as a promising therapy for various types of cancers including lung cancer (Savani et al. 2012; Kieran 2014). Sonic hedgehog (Shh), Smoothened (Smo) and Gli1 are important factors in the SHH signaling pathway.

Polycomb group molecule human Polyhomeotic Homolog 3 (PHC3) is a member of the polycomb Group(PcG) protein family (Robinson et al. 2012), and PcG is a family of chromatin-related gene silencing proteins that regulates gene expression program in epigenetics. PcG proteins have essential roles in early embryonic development and have been implicated in embryonic stem cell pluripotency (Boyer et al. 2006). The PRCs silence tumor-suppressor genes by histone modifications, leading to cancer cell proliferation, metastasis and drug resistance (Crea et al. 2013). Thus, PHC3, similar to SHH signaling pathway, plays an important role in controlling the growth of stem cells in early embryonic development. However, the role of PHC3 and SHH signaling pathways in human lung CSCs still needs to be elucidated.

This study is based on the hypothesis that SFN inhibits the proliferation of lung CSCs by regulating Shh signaling pathway and PHC3. In the present study, SFN can inhibits the proliferation of lung CSCs. And SHH signaling pathway and PHC3 were activated in lung CSCs. Importantly, the results proved that SFN is a potent drug preventing lung CSCs cell growth through regulating Shh signaling pathway and polycomb group molecule PHC3.

\section{Materials And Methods}

\section{Cell culture}

Human non-small cell carcinoma of the lung cancer A549 and H460 cell lines were obtained from the Cancer Institute of Southern Medical University (Guangzhou, China). The cells were maintained in a humidified atmosphere of $5 \% \mathrm{CO}_{2}$ at $37{ }^{\circ} \mathrm{C}$ in DMEM culture medium (Hyclone) with $10 \%$ fetal bovine serum (Gibco). The lung CSCs which express CD133 were isolated by the BD MACs and identified by flow cytometry (FACS). CD133-positive cells were cultured in MEBM basal medium (CBM, New Jersey, USA) to maintain the characteristics of stem cells. 


\section{Cell viability assay}

Cells in logarithmic phase were seeded into 96 -well plates at a cell density of $1 \times 10^{4} /$ well, and then different concentrations of SFN were added into each well and incubated together for $48 \mathrm{~h}$. The final concentrations of SFN were $0,2,4,6,8,10,12 \mu \mathrm{mol} / \mathrm{L}$, respectively. Then, $10 \mu \mathrm{L}$ MTT reagent of concentration $5 \mu \mathrm{g} / \mathrm{L}$ was added into the cell medium of each well and incubated for $4 \mathrm{~h}$ at $37^{\circ} \mathrm{C}$. Following the removal of the supernatant, $150 \mu \mathrm{L}$ dimethyl sulfoxide(DMSO) was added to dissolve formazan. The absorbance at $490 \mathrm{~nm}$ was measured with a microplate reader(Beckman Coulter, Brea, CA, USA). Each reaction was performed in triplicate. At the same time, changes in cell density were observed by optical microscope.

\section{Isolation and identification of lung cancer stem cells}

CD133-positive cells were obtained from A549 and H460 cells using CD133 Microbeads by MiniMACS separator(MiltenyiBiotec, Bergish Gladbach, Germany). A549 and H460 cells were collected separately by centrifugation. Different groups of cells $\left(1 \times 10^{8}\right.$ cells/sample) were resuspended in $500 \mu$ l of the degassed buffer, respectively. Then the cell suspension was added onto the prepared column. The unlabeled cells (CD133-negative) were collected as they passed through the columns. MS columns were washed with degassed buffer. Then the column was removed from the separator and placed on a new suitable collection tube. Buffer was pipetted onto the column, and a fraction was immediately flushed out with the magnetically labeled cells $\triangle C D 133$-positive冈by firmly applying the plunger supplied with the column.

\section{Flow cytometry analysis}

Cells were collected separately by centrifugation, then the cells were washed twice with PBS solution, and up to $1 \times 10^{6}$ cells were resuspended in $500 \mu$ l of PBS, respectively. The cells were then incubated with PE mouse anti-human CD133 for $30 \mathrm{~min}$ at room temperature in the dark. The positive cells were detected using a flow cytometer (BD Biosciences, San Jose, CA, USA) and were analyzed by FlowJo 9.1 software.

\section{TumorSphere formation assay}

Cells were placed in 6-well ultralow attachment plates (Corning Inc.) at a density of 1,000 cells/mL in tumorsphere culture medium DMEM (Invitrogen, Carlsbad, CA, USA) supplemented with 1\% N2 supplement, $2 \%$ B27 supplement, and $100 \mathrm{ng} / \mathrm{mL}$ epidermal growth factor at $37^{\circ} \mathrm{C}$ in a humidified atmosphere of $95 \%$ air and $5 \% \mathrm{CO}_{2}$. These cells were then treated with different concentrations of SFN at the same time. Primary spheroids were collected following 14 days of culture, and tumorspheres were measured using an inverted microscope system (magnification, Eclipse Ti-s, Nikon, Tokyo, Japan).

\section{Reverse transcription and QPCR analysis}

The total RNA was extracted from cells with Trizol reagents. cDNA was synthesized from $1 \mu \mathrm{g}$ of mRNA with a high capacity CDNA reverse transcription kit according to the manufacturer's instructions. Subsequently, cDNA was amplified by qPCR with the SYBR Premix Ex Taq kit according to the 
manufacturer's instructions using the ABI7300 Sequence Detection System. The following gene-specific primers were used: Shh(forward)5'-CGC ACC TGC TCT TTG TGG-3',(reverse)5'-GGA GCG GTT AGG GCT ACT CT-3'; Smo(forward) 5'-TCG CTA CCC TGC TGT TAT TC-3', (reverse) 5'-GAC GCA GGA CAG AGT CTC AT-3'; Gli1(forward) 5'-CTG GAT CGG ATA GGT GGT CT-3', (reverse)5'-CAG AGG TTG GGA GGT AAG GA-3'; PHC3(forward) 5'-AGT GGG GAG AGG AGA AGA-3',(reverse) 5'-GGT GGT GGA ACA GAA ACA-3'. The housekeeping gene Beta-actin was used as a loading control. PCR conditions were as follows: one cycle at $95^{\circ} \mathrm{C}$ for $3 \mathrm{~min}$, followed by 40 cycles at $95^{\circ} \mathrm{C}$ for $30 \mathrm{~s}, 55^{\circ} \mathrm{C}$ for $30 \mathrm{~s}$, and $72^{\circ} \mathrm{C}$ for $1 \mathrm{~min}$. All assays were performed in triplicate and were calculated on the basis of $\Delta \Delta \mathrm{Ct}$ method. The $\mathrm{n}$-fold change in mRNAs expression was determined according to the method of $2^{-\Delta \Delta C T}$.

\section{Western blotting}

Protein sample was extracted from cells with an ice-cold SDS protein lysis buffer. Protein concentration was measured by a Micro BCA Protein Assay Reagent kit. Then protein sample was separated by $10 \%$ SDS-PAGE electrophoresis and transferred onto $0.45 \mathrm{~mm}$ PVDF membranes. The membranes were blocked with $5 \%$ non-fat milk in TBST buffer for $1 \mathrm{~h}$, incubated overnight with primary antibody at $4^{\circ} \mathrm{C}$ and then incubated with secondary antibody for $1 \mathrm{~h}$ at room temperature. The antigen-antibody complexes were visualized using the ECL detection system. The analysis of the bands was conducted by the Image $\mathrm{J}$ software. The following antibodies were purchased from commercial sources including antiShh (ab53281), anti-Smo (ab32575), anti-Gli1(ab134906), anti-PHC3 (GTX32785) and anti-GAPDH (CWBIO).

\section{RNA interference}

siRNAs for SHH were purchased from Invitrogen and the following sequences were used. No.1,SHH-s:5'ACAGGCUGAUGACUCAGAGGUGUAA-3',SHH-as:5'-UUACACCUCUGAGUCAUCAGCCUGU-3';No.2,SHH-s: 5'GGUGUACUACGAGUCCAAGGCACAU-3', SHH-as: 5'-GACUCGUAGUACACC-3'; No.3,SHH-s: 5'CCGACAUCAUAUUUAAGGAUGAAGA-3', SHH-as: 5'-UCUUCAUCCUUAAAUAUGAUGUCGG-3. Cells were seeded in 6-well plates at a cell density of $3 \times 10^{5}$ cells/well in $10 \%$ serum medium without antibiotics. After 24h, cells were transfected in Opti-MEM using LipofectamineRNAiMAX(Invitrogen) and the 20 nmole siRNA was resuspended according to manufacturers instructions. The cells treated with Stealth negative control med component of kit (\#12935300, Invitrogen) were served as controls (si-control).siRNA transfection efficiency in cells was assessed by Western-blotting.

\section{Statistical Analysis}

Statistical analysis was performed using SPSS16.0 software. Data presented were mean \pm SD from three different experiments. Statistical significance between different groups was determined using Students $t$ test. A value of $P<0.05$ was considered statistically significant.

\section{Results}




\section{SFN inhibits the growth of lung cancer cells and lung CSCs}

To explore the role of SFN in lung cancer cells. We first detected cell viability through the MTT assay. The result showed that SFN could inhibit the growth of lung cancer A549 and H460 cells, which was clearly indicated by a declined cell viability trend in a dose-dependent manner following treatment with different concentrations of SFN(Fig. 1A). Electron microscope also shows a significant decrease in the density of A549 and H460 cells following treatments with $8 \mu \mathrm{mol} / \mathrm{L} \mathrm{SFN} \mathrm{for} \mathrm{48h} \mathrm{(Fig.} \mathrm{1B).}$

To investigate whether SFN sensitized to lung CSCs, we first isolated CD133 + cells from the human lung cancer A549 and H460 cells by MACS. CD 133 + cells accounted for $96.4 \%$ and $97.8 \%$ in A549 and H460 cells after magnetic sorting (Fig. 1C,D). The CD133 + cells can form a colony in the tumorsphere culture medium. In sphere formation assay, the A549/CD133 + and H460/CD133 + cells were treated with 0, 4, 8 and $12 \mu \mathrm{mol} / \mathrm{L} \mathrm{SFN}$. At the end of 14 days, the volume of tumorspheres were measured, and the volume of tumorsphere trend showed a gradual decrease with increasing dose of SFN (Fig. 1E,F). These results showed that SFN can effectively inhibit the proliferation of lung LSCs .

\section{High expression of SHH signaling pathway and PHC3 in lung CSCs}

It has been reported that the SHH signaling pathways and Polycomb group molecule PHC3 play an important role in the control of stem cell growth[7, 14]. However, the role of the SHH signaling pathways and PHC3 in lung CSCs is uncertain. As shown in Fig. 2A,B, the mRNA level of the important components of the SHH signaling pathway such as Shh, Smo and Gli1 were markedly increased in human lung CSCs (CD133+/A549 and CD133+/H460 cells) compared to that in human non-lung stem cells (CD133-/A549 and CD133-/H460 cells) $(P<0.01)$. We also measured PHC3 expression level in CD133 positive cells and CD133 negative cells. PHC3 mRNA expression level was also markedly increased in CD133 positive cells (compare to CD133-negtive cells, $P<0.01$ ).

To further determine whether the SHH signaling pathways and PHC3 have an abnormal expression in human lung CSCs, we examined the protein expression level of Shh, Smo, Gli1 and PHC3 in CD133positive cells and in CD133-negative cells. Interestingly, Shh, Smo, Gli1 and PHC3 protein expression levels were consistent with mRNA transcription level(Fig. 2C-E). These data indicated that Shh signaling pathway and Polycomb group molecule PHC3 were abnormally activated in lung cancer stem cells.

\section{SFN regulates SHH signaling pathway and PHC3 in lung CSCs}

To further explore whether SFN regulates SHH signaling pathway and $\mathrm{PHC} 3$ in lung CSCs, we examined the effects of SFN on the mRNA expression level of Shh, Smo, Gli1 and PHC3 in CD133-positive. As shown in Fig. 3A,B, SFN could markedly inhibit the mRNA expression of Shh, Smo, Gli1 and PHC3 in CD133-positive cells $(P<0.01$ or $P<0.001)$. The expression of these components was also further confirmed by western blotting assay. SFN could also inhibit protein expression of Shh, Smo, Gli1 and PHC3 in the lung cancer stem cells $(P<0.01$ or $P<0.001)$, as shown in Fig. 3C-E. These data suggested that SFN can regulate by SHH signaling pathway and PHC3 in lung CSCs. 


\section{SFN regulates proliferation of lung CSCs through SHH Signaling Pathway and PHC3}

In order to further identify the role of the SHH signaling pathway in the proliferation of lung CSCs, we silenced the Shh gene in CD133+/A549 and CD133+/H460 cell lines. Cells were transfected with siRNAs and maintained for $48 \mathrm{~h}$. The transfection efficiency was determined by western blotting. The Shh protein expression level in siRNA-shh transfected cells was suppressed by approximately 85\% in A549/CD133 + cells $(P<0.001)$ and $94 \%$ in H460/CD133 + cells $(P<0.001)$ compared with the control(Fig. 4A-C).

When the Shh gene was silenced successfully, cell viability of siRNA-shh transfected cells became lower than that of the si-control group. And the number of cells in siRNA-shh group were lower than that of in sicontrol group (Fig. 4F). Further research revealed that the inhibitory effect of SFN on cell proliferation still exists in siRNA-shh cells. The cell viability also showed a gradual decrease with increasing dose of SFN both in siRNA-Shh group and si-control group. But, the decreasing trend of cell viability in siRNA-shh group was significantly less than that in si-control group (Fig. 4D,E). At the same time, the number of cells decreased in both siRNA-shh group and si-control group, but the decrease in siRNA-shh group was weaker than that in si-control group(Fig. $4 \mathrm{~F})$. These results indicate that downregulation of Shh attenuates the inhibitory effect of SFN on proliferation of lung CSCs.

As no prior report on the relationship between $\mathrm{SHH}$ and $\mathrm{PHC} 3$ in lung cancer exists, we employed a further study expression of PHC3 in siRNA-shh transfected cells. Interestingly, PHC3 expression level showed a significant decrease in siRNA-shh cells compared with the control cells $(P<0.01)$ (Fig. 4A-C). These data confirmed that there was a cross link between $\mathrm{SHH}$ signaling pathway and $\mathrm{PHC}$, and both of which were involve in proliferation of lung cancer.

\section{Discussion}

A series of scientific studies have shown that SFN can induce apoptosis in a variety of human cancers (Mi et al. 2009), leading to cell cycle arrest and inhibition of malignant growth. SFN is an isothiocyanate (ITC) extracted from cruciferous vegetables. In our study, we observed that SFN can inhibit the activity of lung cancer stem cells.

CD133 positive cells possess the characteristics of lung CSCs. So, CD133 is an important marker of CSCs (Pavon et al. 2018; Kim et al. 2019). Therefore, we separated lung CSCs which express CD133-positive from the human lung cancer A549 and A460 cells. To investigate whether SFN affects lung CSCs, we performed the sphere formation assay. We observed that the volume of tumorspheres were gradually decreased with the increasing dose of SFN. This result indicates that SFN has the potential to inhibit the proliferation of lung CSCs. However, the mechanism of the phenomenon still needs to be elucidated.

In recent years, several studies have reported that the $\mathrm{SHH}$ signaling pathway plays a critical role in the development and progression of lung cancer (Yang et al. 2018; Giroux et al. 2018; Maitah et al. 2011). It has also been reported that the $\mathrm{SHH}$ signaling pathway could regulate self-renewal and proliferation of CSCs and increase tumor invasiveness(Zhu et al. 2017). In this study, we have demonstrated that the 
$\mathrm{SHH}$ signaling pathway was abnormally activated in lung CSCs, suggesting that the hyperactive $\mathrm{SHH}$ signaling may regulate the expression of stemness genes in lung CSCs and play important roles in the cell proliferation and progression of lung CSCs. Furthermore, when the Shh gene was silenced successfully, the proliferation ability of siRNA-shh cells was decreased as compared to that of the sicontrol group. Therefore, we speculate that the SHH signal pathway plays an important role in the proliferation of lung CSCs. To explore whether SFN inhibits the proliferation of lung CSCs through the $\mathrm{SHH}$ signaling pathway, we further investigated the expression of key components in the $\mathrm{SHH}$ signaling pathway in cells which were treated by SFN. The results showed that SFN can obviously reduce the mRNA and protein expression of Shh, Smo and Gli1 in CD133-positive cells.

Furthermore, cell viability assay showed that the inhibitory effect of SFN on cell proliferation in the siRNAshh cells was much weaker than that in the si-control cells. This work demonstrated that the actions of SFN to inhibit proliferation of lung CSCs is achieved by regulating $\mathrm{SHH}$ signaling pathway.

PHC3 is one of the members of PRCs, which have essential roles in early embryonic development and have been implicated in embryonic stem cell pluripotency (Pachano et al. 2019; Kloet et al. 2016). It has been reported that PHC1 was important in regulating stem cells(Hou et al. 2019; Linxweiler et al. 2012). Other previous studies have shown that PcG complexes control cellular proliferation and favor tumorigenesis(Chan et al. 2019; Wang et al. 2015). AM Iwata et al. (2010) reported that PHC3 expression was abnormal in osteosarcoma. In this study, we investigated whether PHC3 like PHC1 plays the same role in regulating stem cells. Our data indicated that both the mRNA and protein expressions of PHC3 were also markedly increased in human lung CSCs. Further research also showed that SFN inhibited the mRNA and protein expressions of PHC3 in CD133-positive cells. Moreover, PHC3 presented the same expression pattern as SHH signaling pathway in lung CSCs. However, there was no evidence to support the interaction between SHH and PHC3. Therefore, we investigated the protein expression of PHC3 in Shh silenced cells. Here, we demonstrated that downregulation of Shh suppressed PHC3 protein expression in A549 /CD133 + and H460 /CD133 + cells. Together, these data confirmed that there was a cross link between Shh and PHC3, and both Shh signaling and PHC3 were involve in proliferation of lung CSCs. Collectively, these data indicate that $\mathrm{SHH}$ signaling pathway and $\mathrm{PHC}$ play important roles in regulatory effect of SFN in proliferation of lung CSCs.

In conclusion, this study provides evidence that SFN can serve as a potent anticancer agent, inhibit the proliferation of lung CSCs. Furthermore, this study demonstrates that SHH signaling pathway and PHC3 work together in lung CSCs and aberrant activation of these signals promotes tumorigenesis and progression of lung cancer. These findings suggested that SFN could be exploited in a novel therapeutic avenue for lung cancer treatment by regulating the $\mathrm{SHH}$ signaling pathways and $\mathrm{PHC}$.

\section{Declarations}

\section{Acknowledgements}

Special thanks are given to M.D. Jiateng Zhong for his dedicated revision of the manuscript. 


\section{Authors' contributions}

This work was conceived and designed by FW and PL. FW carried out the data analysis and wrote the manuscript. The experiments were carried out by $\mathrm{YS}, \mathrm{XH}, \mathrm{CQ}$ and $\mathrm{WZ}$. MW are responsible for re-analyzing data and revision. All authors read and approved the final manuscript.

\section{Funding}

This work was supported by the National Natural Science Foundation of China (81803607), Foundation for University Key Teacher of Henan Province (2018GGJS101), Key Scientific and Technological Projects of Henan Province (202102310032), Science and Technology Innovative Research Team in Higher Educational Institutions of Henan Province (20IRTSTHN030), Natural Science Foundation of Henan Province (212300410013). The funding agency was not involved in the design of the study and collection, analysis, and interpretation of data and in writing the manuscript.

\section{Availability of data and material}

The datasets used and/or analyzed during this study are available from the corresponding author on reasonable request.

\section{Ethics approval and consent to participate}

Cell lines used in this study, did not require ethical approval.

\section{Consent for publication}

Not applicable.

\section{Competing interests}

Authors declare that they have no conflict of interest

\section{References}

1. Torre LA, Siegel RL, Jemal A (2016) Lung Cancer Statistics. Adv Exp Med Biol 893:1-19.

2. Siegel RL, Miller KD, Jemal A (2019) Cancer statistics, 2019. CA Cancer J Clin 69:7-34.

3. Bray F, Ferlay J, Soerjomataram I, Siegel RL, Torre LA, Jemal A (2018) Global cancer statistics 2018: GLOBOCAN estimates of incidence and mortality worldwide for 36 cancers in 185 countries. CA Cancer J Clin 68:394-424.

4. Hermann PC, Bhaskar S, Cioffı M, Heeschen C (2010) Cancer stem cells in solid tumors. Semin Cancer Biol 20:77-84.

5. Kim WT, Ryu CJ (2017) Cancer stem cell surface markers on normal stem cells.BMB Rep 50:285-298. 
6. Mizugaki H, Sakakibara-Konishi J, Kikuchi J, Moriya J, Hatanaka KC, Kikuchi E (2014)CD133 expression: a potential rognostic marker for non-small cell lung cancers. Int J ClinOncol 19:254-259.

7. Clarke JD, Dashwood RH, Ho E (2008) Multi-targeted prevention of cancer by sulforaphane. Cancer Lett 269: 291-304.

8. Sangthong S, Weerapreeyakul N (2016) Simultaneous quantification of sulforaphene and sulforaphane by reverse phase HPLC and their content in Raphanussativus L. var. caudatusAlef extracts. Food Chemistry 181:139-144.

9. Fisher ML, Ciavattone N, Grun D, Adhikary G, Eckert RL (2017) Sulforaphane reduces YAP/Np63alpha signaling to reduce cancer stem cell survival and tumor formation. Oncotarget 8:73407-73418.

10. Jaman MS, Sayeed MA (2018) Ellagic acid, sulforaphane, and ursolic acid in the prevention and therapy of breast cancer: current evidence and future perspectives. Breast Cancer 25:517-528.

11. Srivastava RK, Tang SN, Zhu W, Meeker, Shanka S (2011) Sulforaphane synergizes with quercetin to inhibit self-renewal capacity of pancreatic cancer stem cells. Front Biosci (Elite Ed) 3:515-528.

12. Subramaniam D, Kaushik G, Dandawate P, Anant S (2018) Targeting Cancer Stem Cells for Chemoprevention of Pancreatic Cancer. Curr Med Chem 22:2585-2594.

13. Varjosalo M, Taipale J (2008) Hedgehog: functions and mechanisms. Genes Dev22: 2454-2472.

14. Subramani R, Gonzalez E, Nandy SB, Arumugam A, Camacho F, Medel J, Alabi D, Lakshmanaswamy R. (2017) Gedunin inhibits pancreatic cancer by altering sonic hedgehog signaling pathway.Oncotarget 14:10891-10904.

15. Song Z, Wei B, Lu C, Huang X, Li P, Chen L (2017) Metformin suppresses the expression of Sonic hedgehog in gastric cancer cells. Mol Med Rep 15:1909-1915.

16. Savani M, Guo Y, Carbone DP, Csiki I (2012) Sonic hedgehog pathway expression in non-small cell lung cancer. TherAdv Med Oncol 4: 225-233.

17. Kieran MW (2014) Targeted treatment for sonic hedgehog-dependent medulloblastoma. Neuro Oncol 16:1037-1047.

18. Robinson AK, Leal BZ, Nanyes DR, Kaur Y, llangovan U, Schirf V, Hinck AP, Demeler B, Kim CA (2012). Human polyhomeotic homolog 3 (PHC3) sterile alpha motif (SAM) linker allows open-ended polymerization of PHC3 SAM. Biochemistry 51: 5379-5386.

19. Boyer LA, Plath K, Zeitlinger J, Brambrink T, Medeiros LA, Lee TI, Levine SS, Wernig M, Tajonar A, Ray MK, Bell GW, Otte AP, Vidal M, Gifford DK, Young RA, Jaenisch R (2006) Polycomb complexes repress developmental regulators in murine embryonic stem cells. Nature 18:349-353.

20. Crea F, Sun L, Pikor L, Frumento P, Lam WL, Helgason CD (2013) Mutational analysis of Polycomb genes in solid tumours identifies PHC3 amplification as a possible cancer-driving genetic alteration. Br J Cancer 109: 1699-1702.

21. Mi L, Gan N, Cheema A, Dakshanamurthy S, Wang X, Yang DC, Chung FL (2009) Cancer preventive isothiocyanates induce selective degradation of cellular alpha- and beta-tubulins by proteasomes. $\mathrm{J}$ Biol Chem 284: 17039-17051. 
22. Pavon LF, Sibov TT, de Souza AV, da Cruz EF, Malheiros SMF, Cabral FR (2018) Tropism of mesenchymal stem cell toward CD133(+) stem cell of glioblastoma in vitro and promote tumor proliferation in vivo. Stem Cell Res Ther 9:310.

23. Kim TM, Ko YH, Ha SJ, Lee HH (2019) Impact of in vitro driven expression signatures of CD133 stem cell marker and tumor stroma on clinical outcomes in gastric cancers. BMC Cancer 19:119.

24. Yang B, Miao S, Li Y (2018) SCUBE2 inhibits the proliferation, migration and invasion of human nonsmall cell lung cancer cells through regulation of the sonic hedgehog signaling pathway. Gene 672:143-149.

25. Giroux-Leprieur E, Costantini A, Ding VW, He B (2018) Hedgehog Signaling in Lung Cancer: From Oncogenesis to Cancer Treatment Resistance. Int J Mol Sci 9:2835.

26. Maitah MY, Ali S, Ahmad A, Gadgeel S, Sarkar FH(2011) Up-regulation of sonic hedgehog contributes to TGF-beta1-induced epithelial to mesenchymal transition in NSCLC cells. PLoS One 13:e16068.

27. Zhu JY, Yang X, Chen Y, Jiang Y, Wang SJ, Li Y,Wang XQ, Meng Y, Zhu MM, Ma X, Huang C, Wu R, Xie CF, Li XT Geng,SS, Wu JS, Zhong CY, Han HY (2017).Curcumin Suppresses Lung Cancer Stem Cells via Inhibiting Wnt/beta-catenin and Sonic Hedgehog Pathways. Phytother Res31:680-688.

28. Pachano T, Crispatzu G, Rada-Iglesias A (2019) Polycomb proteins as organizers of 3D genome architecture in embryonic stem cells. Brief Funct Genomics 18:358-366.

29. Kloet SL, Makowski MM, Baymaz HI, van Voorthuijsen L, Karemaker ID, Santanach A, Jansen P, Croce L D, Vermeulen M (2016) The dynamic interactome and genomic targets of Polycomb complexes during stem-cell differentiation. Nat Struct Mol Biol 23:682-690.

30. Hou J, Wang X (2019) The polycomb group proteins functions in epithelial to mesenchymal transition in lung cancer. Semin Cell Dev Biol 90:138-143.

31. Linxweiler M, Linxweiler J, Barth M, Benedix J, Jung V, Kim YJ, Bohle RM, Zimmermann R, Greiner M (2012) Sec62 bridges the gap from $3 q$ amplification to molecular cell biology in non-small cell lung cancer. Am J Pathol 180:473-483.

32. Chan HL, Morey L (2019) Emerging Roles for Polycomb-Group Proteins in Stem Cells and Cancer. Trends Biochem Sci 44:688-700.

33. Wang W, Qin JJ, Voruganti S, Nag S, Zhou J, Zhang R (2015) Polycomb Group (PcG) Proteins and Human Cancers: Multifaceted Functions and Therapeutic Implications. Med Res Rev 35:1220-1267.

34. Iwata S, Takenobu H, Kageyama H, Koseki H, Ishii T, Nakazawa A, Tatezaki S, Nakagawara A, Kamijo T (2010) Polycomb group molecule PHC3 regulates polycomb complex composition and prognosis of osteosarcoma. Cancer Sci101: 1646-1652.

\section{Figures}


A

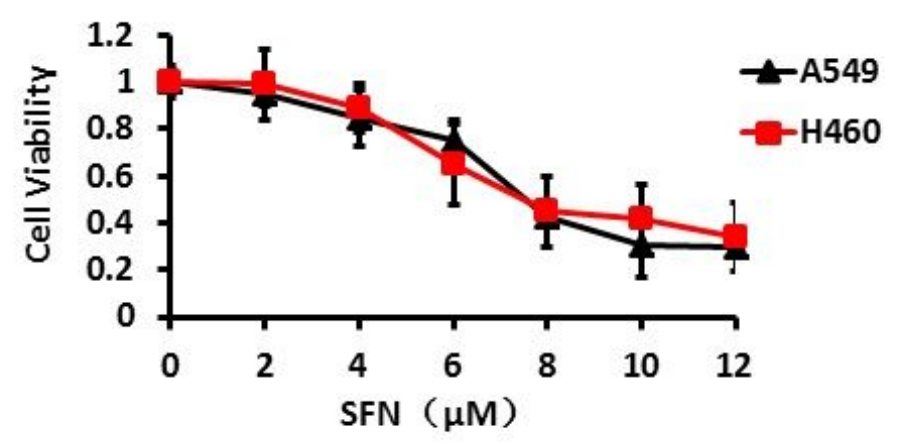

C
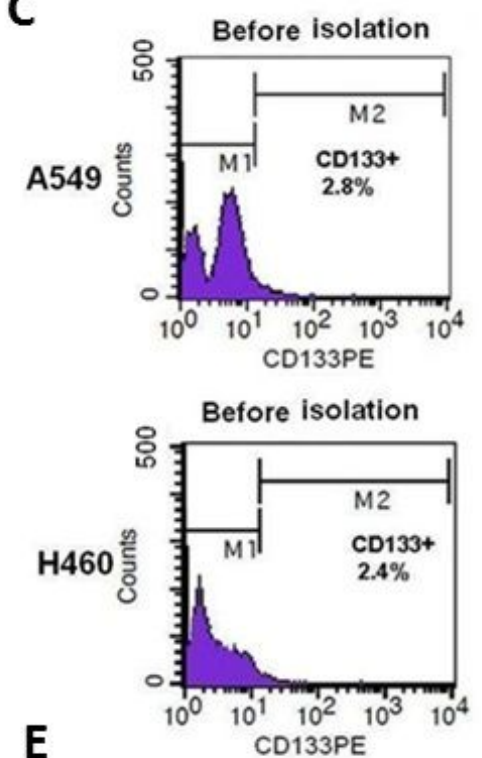

E
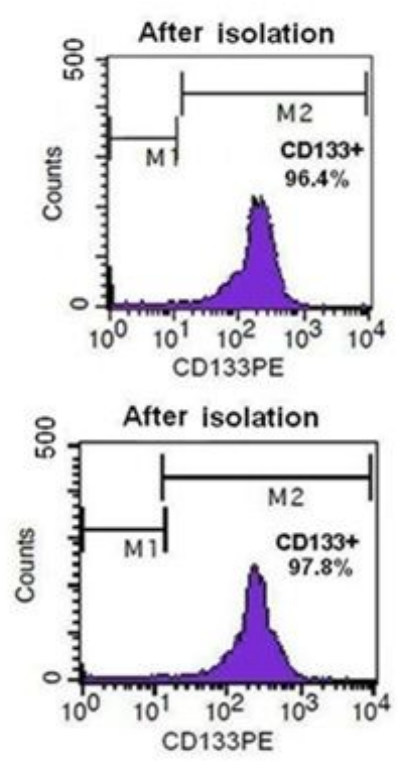

B

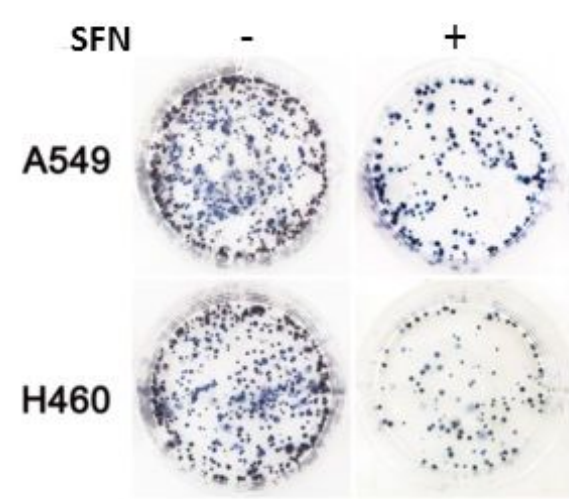

D
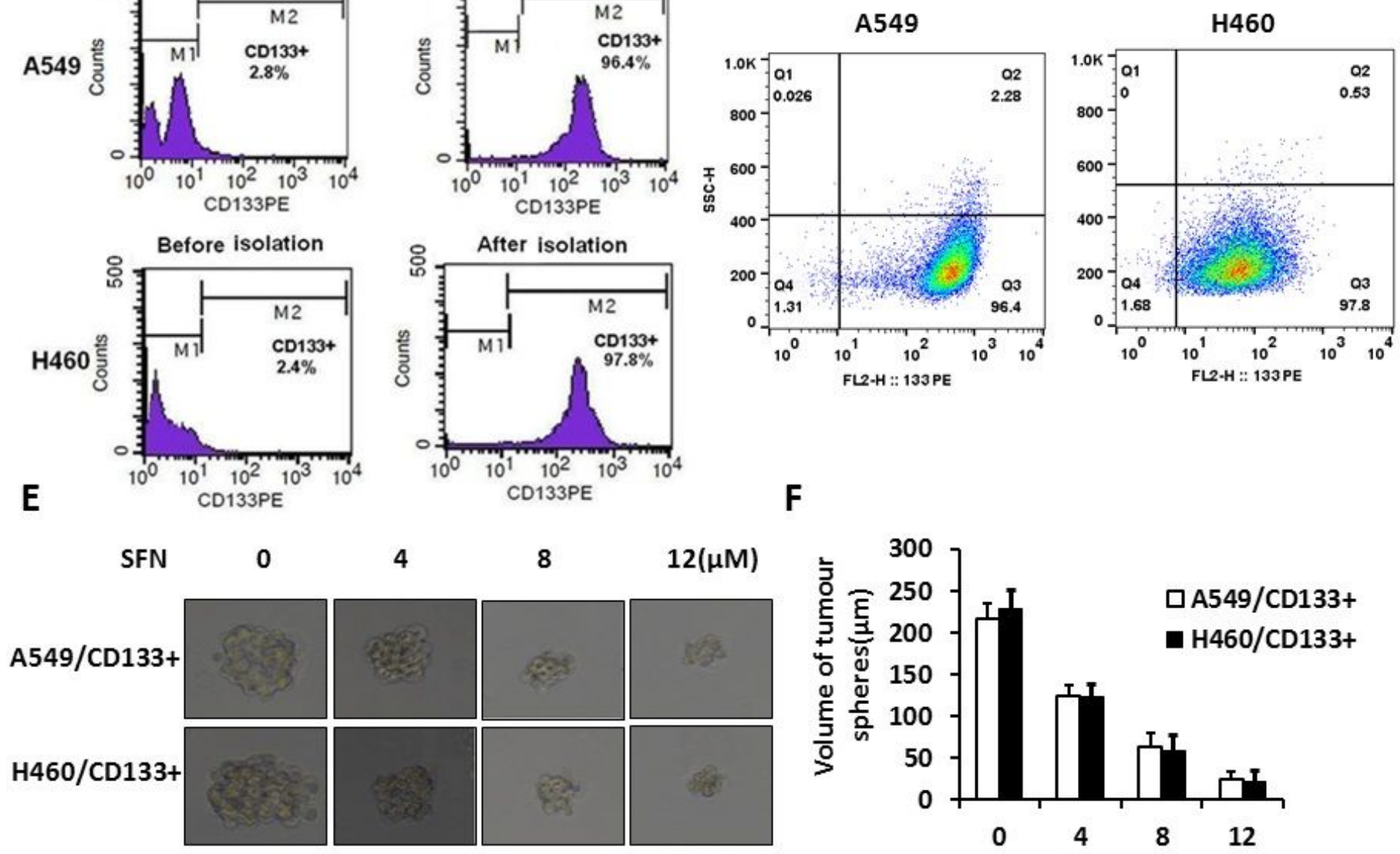

4

8

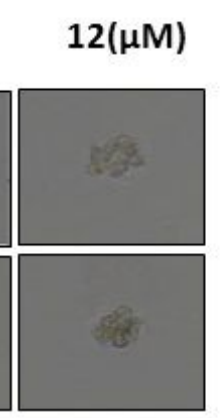

\section{Figure 1}

Inhibitory effect of SFN on cell proliferation. (A) Inhibitory effect of SFN on viability of A549 and H460 cells in a dose-dependent manner following treatments with $0,2,4,6,8,10$ and $12 \mu \mathrm{mol} / \mathrm{L} \mathrm{SFN}$ for $48 \mathrm{~h}$ by MTT assay. (B) Inhibitory effect of SFN on cell density of A549 and H460 cells following treatments with $8 \mu \mathrm{mol} / \mathrm{L}$ SFN for $48 \mathrm{~h}$ by optical microscope. (C,D) CD133 positive cells were isolated from human lung cancer A549 and H460 cells by Macs. Before sorting, the positive rate of CD133 are $2.8 \%$ and $2.4 \%$. And after sorting, the positive rate of CD133 are $96.4 \%$ and $97.8 \%$ in A549 cells and H460 cells, respectively. 
(E,F) The tumor sphere volume of A549/CD133+ and H460/CD133+ cells were inhibited by SFN in a dosedependent manner.

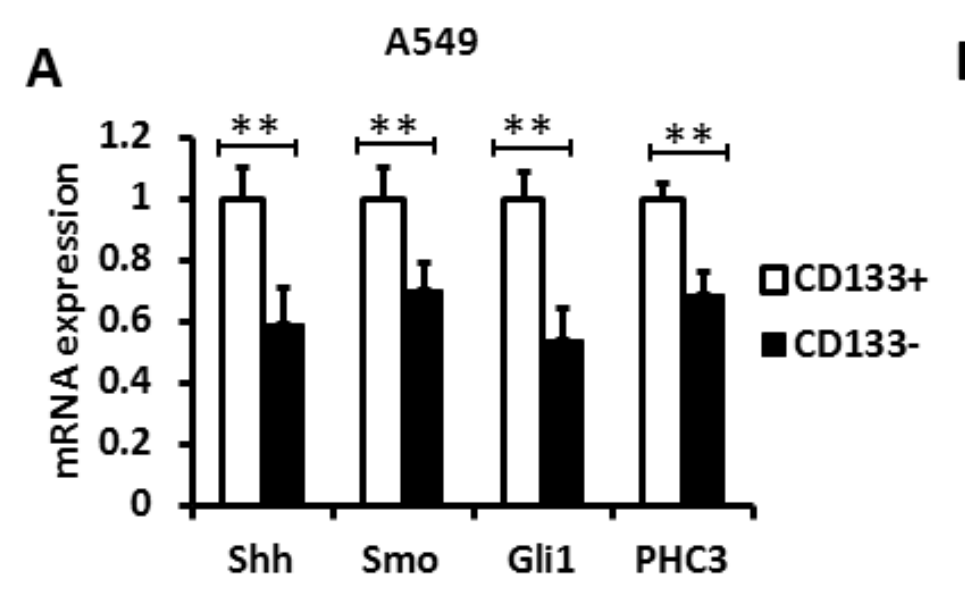

B

H460

C

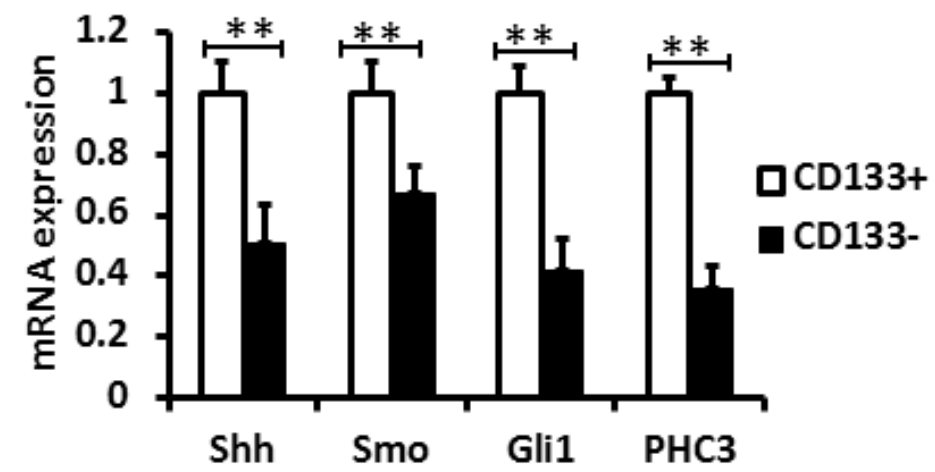

D
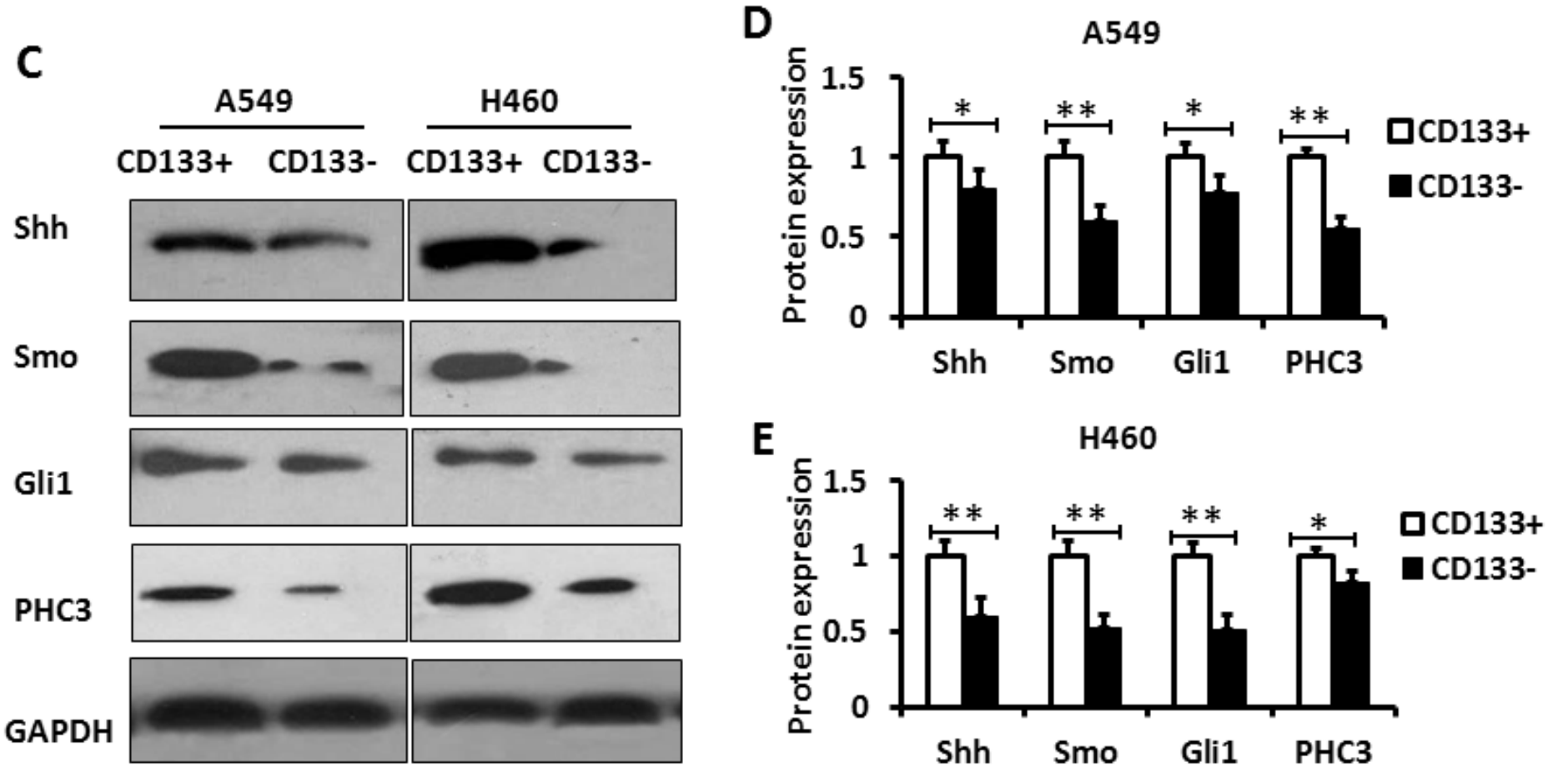

E

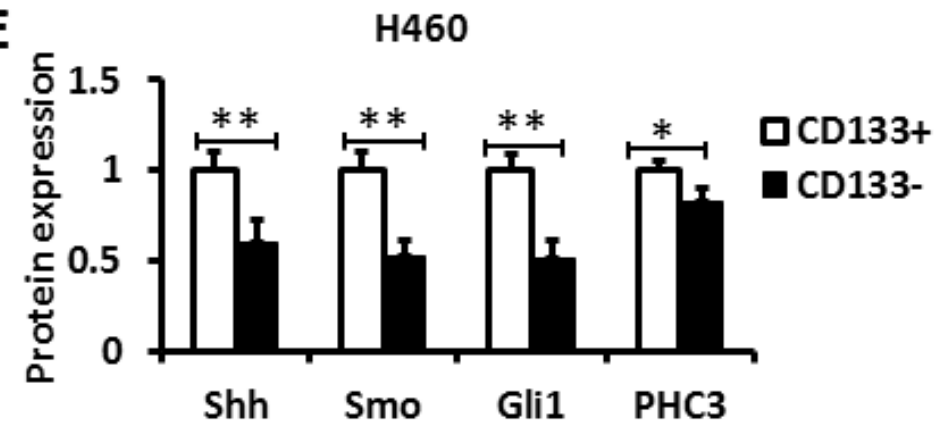

Figure 2

Expression of Shh, Gli1, Smo and PHC3 in CD133+ lung CSCs and in CD133- lung cancer cells. $(A, B)$ QPCR data shows that the mRNA levels of Shh, Gli1, Smo and PHC3 in CD133+/A549 and CD133+/H460 are higher than those in CD133-/A549 and CD133-/H460. (C-E) Western blotting data shows that the protein levels of Shh, Gli1, Smo and PHC3 in CD133+/A549 and CD133+/H460 cells are higher than those in CD133-/A549 and CD133-/H460 cells. The relative protein levels were quantified with Image $J$ software. $N=3$, Data are presented as mean $\pm S D$, ${ }^{*}<<0.05,{ }^{\star} * P<0.01,{ }^{\star *} * \mathrm{P}<0.001$. 

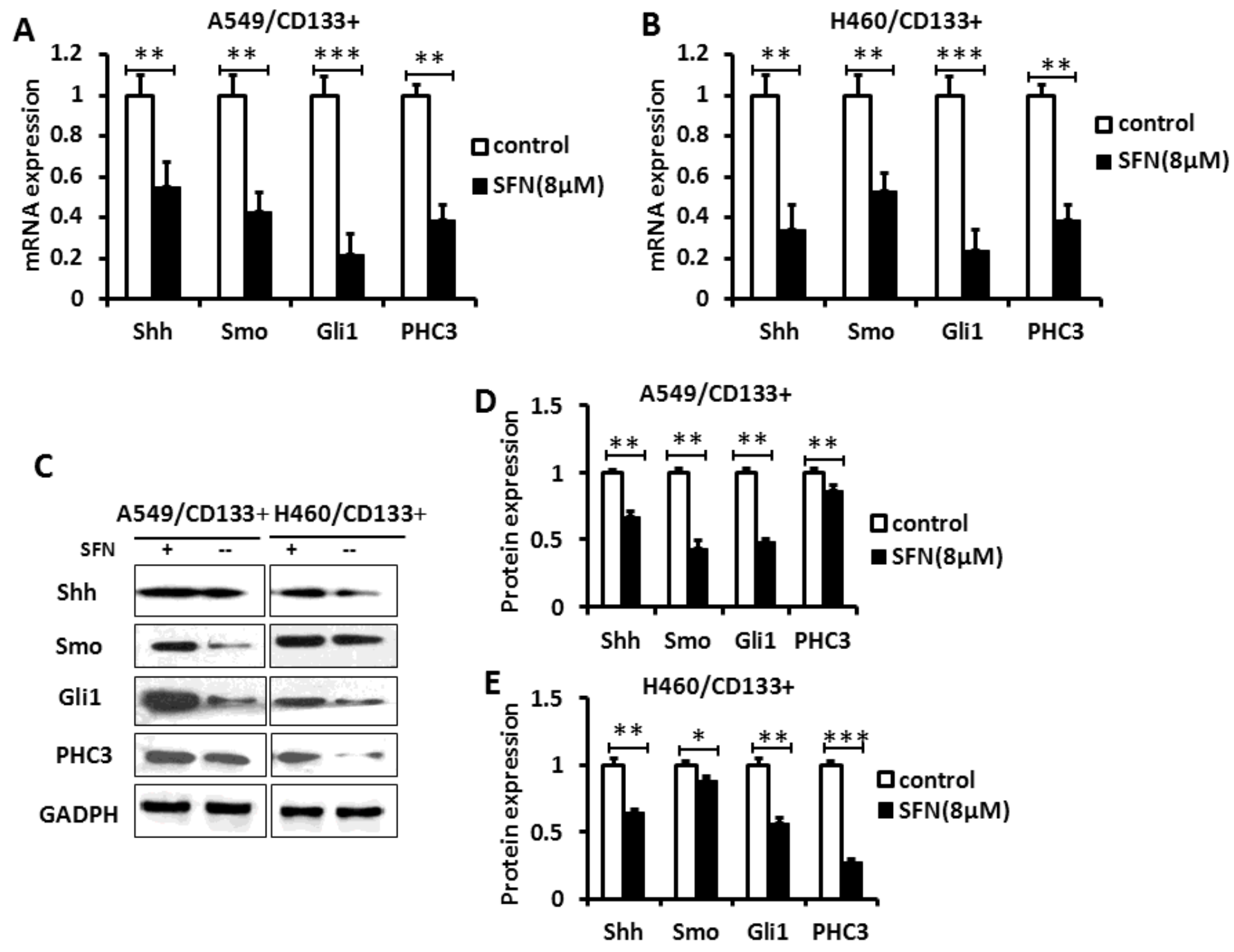

Figure 3

SFN inhibits expression of Shh, Gli1, Smo and PHC3 in human lung CSCs. (A,B) QPCR data shows that the mRNA levels of Shh, Gli1, SMO and PHC3 were significantly inhibited when lung CSCs (CD133+/A549 and $\mathrm{CD} 133+/ \mathrm{H} 460$ cells)were treated with SFN,compared with the control group. (C-E) Western blotting data shows that the protein levels of Shh, Gli1, SMO and PHC3 were significantly inhibited when lung CSCs (CD133+/A549 and CD133+/H460 cells)were treated with SFN,compared with the control group. N $=3$, Data are presented as mean $\pm S D, * P<0.05, \star \star P<<0.01, * \star * P<0.001$. 
A

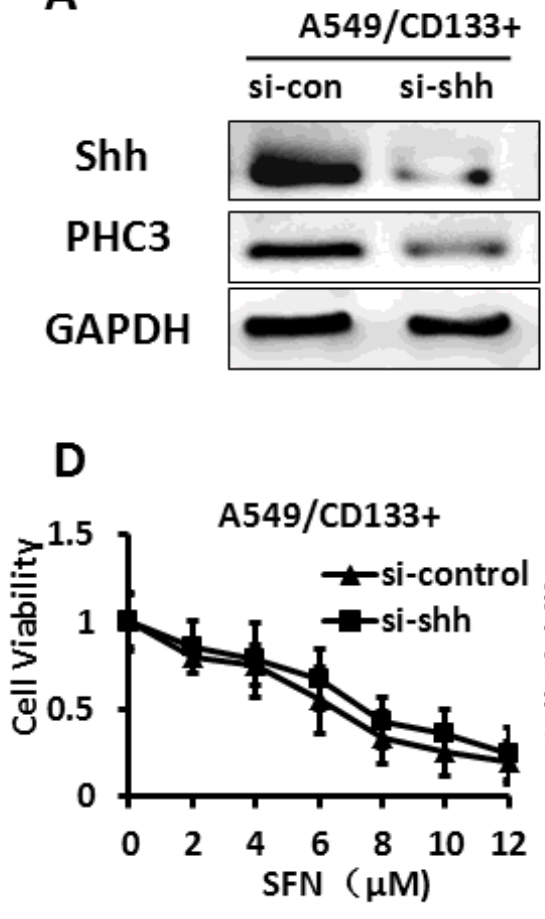

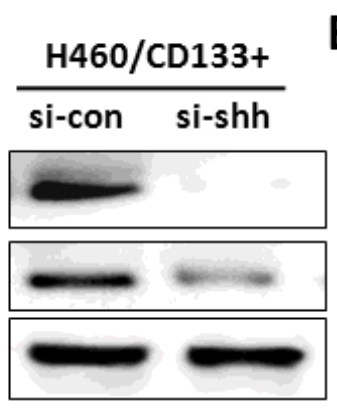

E
B

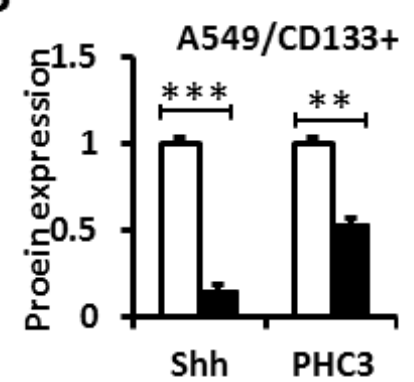

C

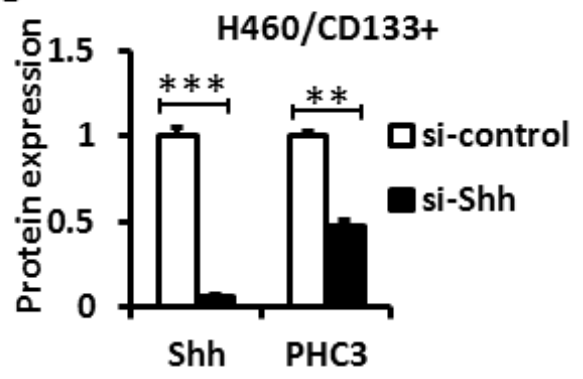

$\mathbf{F}$
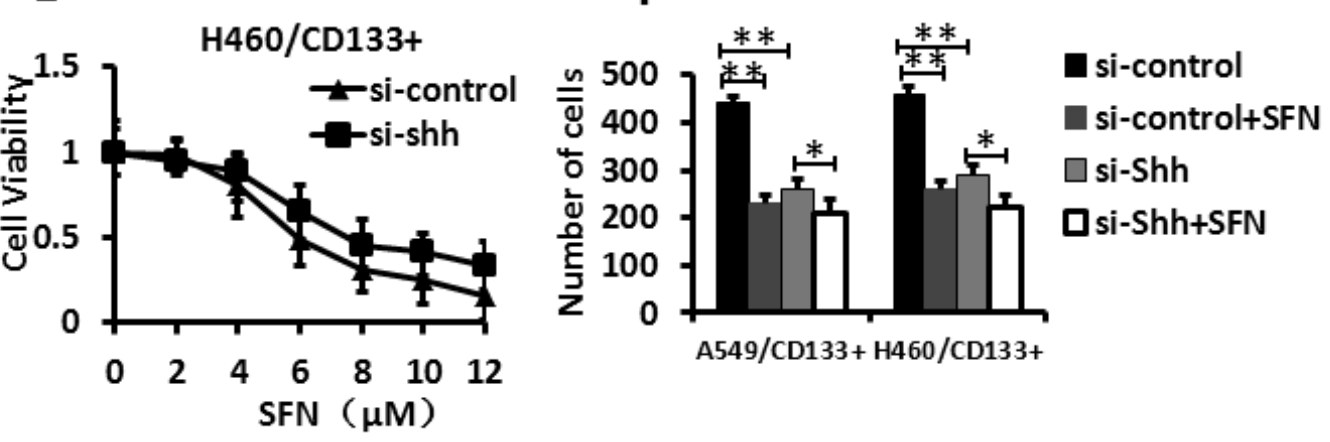

Figure 4

Expression of PHC3, Shh and cell proliferation after knockdown shh. (A-C) The efficiency of siRNA to silence Shh gene were determined by Western blotting following $48 \mathrm{~h}$ transfection. And the expression of Shh is suppressed to $15 \%$ in CD133+/A549 and 6\% in CD133+/H460 cells, respectively. And the expression of PHC3 is suppressed to 53\% in CD133+/A549 and 47\% in CD133+/H460 cells, respectively. The relative protein levels were quantified with Image $\mathrm{J}$ software. $(D, E)$ In the cell proliferation assay, the cell viability shows a gradual decrease with increasing dose of SFN both in siRNA-shh group and sicontrol group. Furthermore, the decreasing trend of siRNA-shh group are less than that of si-control group with increasing dose of SFN. (F) Cell numbers of siRNA-shh cells are significantly less than that of sicontrol group following $48 \mathrm{~h}$ transfection. And the inhibitory effect of SFN on siRNA-Shh cells are significantly lower than that of control group. $N=3$, Data are presented as mean $\pm S D, * P<0.05$, $* * P<$ $0.01, * \star * P<0.001$. 